\title{
Application of PODEM Algorithm for Fault Detection and Location in FinFet based Combinational VLSI Circuits
}

\author{
K.V.B.V Rayudu, D R Jahagirdar, P Srihari Rao
}

\begin{abstract}
FinFet transistors are used in major semiconductor organizations and a significant role is played by it in developing the silicon industries. Due to few embedded memories and other circuit issues the transistors have specific faults in manufacturing, designing of the circuit etc. This paper presents an advanced test algorithm to diagnose those faults. The circuit with different gates is designed to identify the places having faults. In addition, different algorithms such as PODEM (Path Oriented Decision Making algorithms) are used to find the fault detection and location. The Furthermore, more complicated circuits are analyzed for fault detection with different approach. In this research work Combinational Circuits are designed using $20 \mathrm{~nm} / 32 \mathrm{~nm}$ technology nodes in LT Spice environment and PODEM Algorithm is implemented which is developed in MATLAB, to detect and identify fault location and sensitive test vector to detect fault in the circuit and results are presented..
\end{abstract}

Keywords: Fin Fet transistors, Fault analysis, Transfer characteristics. , PODEM Algorithm, Fault Diagnosis, Fault Detection.

\section{INTRODUCTION}

The MOS transistor models are highly complex as the dimensions of devices are decreasing day by day. The model Parameters which is required for the circuit simulations needs to be extracted successfully. Alternate use of MOSFETs is the circuits involving the FinFet which is being investigated. The gate control over the channel is better when compared to MOSFETs ${ }^{1}$.FinFETs are defined as the circuits with the shape of fins and is perpendicular to the structure of wafer in which the current is transferred. The existing fin will be sandwiched in-between front as well as back gates. (Ref: Fig 1) In order to suppress short channel effect, the structures of FinFETs are very thin ${ }^{2}$. Fins are compact and sophisticated such that its size is less than the channel length. The traditional use of the planar bulk equipment of sub $25 \mathrm{~nm}$ with short channel behaviour, reduced leakage current and

Revised Manuscript Received on December 30, 2019.

* Correspondence Author

K.V.B.V Rayudu, Scientist 'G' Head, Reliability Engineering Division, Research Centre Imarat, Vignyanakancha Po, Hyderabad, India-500069. D R Jahagirdar, Scientist ' $G$ ' Research Centre Imarat, Vignyana Kancha Po, Hyderabad, India-500069.

Dr P Srihari Rao, Associate Professor, NIT Warangal, Telangana, India-506004.

(C) The Authors. Published by Blue Eyes Intelligence Engineering and Sciences Publication (BEIESP). This is an open access article under the CC BY-NC-ND license (http://creativecommons.org/licenses/by-nc-nd/4.0/) excellent fabrication process is replaced by the double gate FinFet transistors which remain considered as the worst substitute. By adopting simple manufacturing process and having goodcompatibility with planar MOSFET, FinFet is considered as one of the most feasible multi-gate devices ${ }^{3}$.

Numerous The conventional MOSFET is used for the scaling of power which is an issue due to short channel effect 4 .

There are numerous types of fault models available for FinFETS ${ }^{5}$.There are few problems associated with the design of FinFet circuits in various applications. At various levels of abstraction, fault modelling for the planar single gate

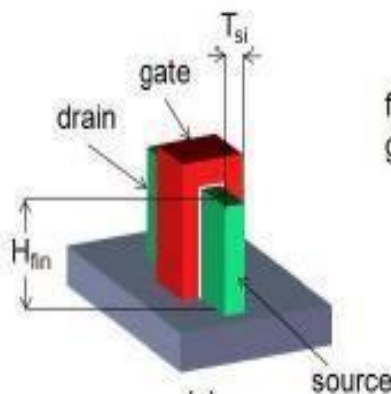

(a)

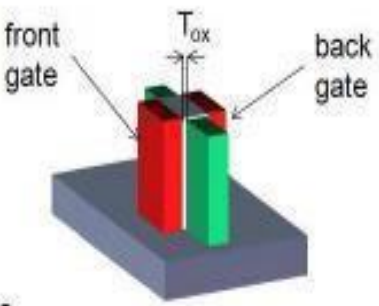

(b)
Figure1.A) Shorted gate B) Independent gate FinFETs.

CMOS is researched extensively ${ }^{6}$. Considering an example of bridging, stuck-open faults and stuck at delay faults are the broadly utilized fault models in CMOS. Several defects such as resistive shorts and open are found because of the improvements in the technology. The technology advancement also leads to the process variability of manufactured circuits. The process variability makes the interconnected parameters modelled as random variables. The uncertainty is captured by the random variables by the process variability, change in the environment conditions such as temperature, voltage etc.

\section{RELATED WORK AND LITERATURE SURVEY}

1. The specific faults in FinFet circuits with a new strategy are presented ${ }^{8}$. Further the fault modelling is performed and besides that, test algorithm which is novel is proposed for synthesis. The validation isperformed on the other FinFet based embedded technologies. Faults specific to the FinFETs are identified.

2. The work is based on the model of FAST fault and is proposed on behalf of the insignificant delay faults by the cross-gate defects in FinFET ${ }^{9}$. 
The test patterns are generated as well as selected using FAST ATPG, test selection and fault simulation. The comparison is made between the FAST SDQL and FAST coverage and the pattern sets which are obtained are $29 \%$ and $4 \%$ respectively.

3. The problems associated with the fault diagnosis are overcome by an improved partial least square approach $(\text { IPLS })^{10}$. The IPLS helps in decomposing the process variables with the key performance indicator related part. Fault diagnosis information is obtained by the test statistics which are designed and the desired performance of the systems is presented. The proposed approach helps in diagnosing KPI faults and also provides the high fault detection rate.

4. A new approach is presented to find the internal cell delay effects using the analog simulation-based fault models 11 .

5. All the defects present in the FinFet circuits are modelled by the CMOS fault models. Mixed-mode Centaurs TCAD device is used for the simulation of the problems which are present in the circuit and shows that the faults overlap in a planar MOSFET and FINFET ${ }^{12}$. New faults are required to capture the behaviour of the logic gates.

6 . The sequential test generation algorithm which is more effective for the sequential circuits is implemented ${ }^{13}$. The standard PODEM algorithm using $\mathrm{C}++$ which is fully tested using combinational circuits is proposed. The results show that test patterns on behalf of line stuck-at faults may be generated for the circuits.

7. To overcome the inefficiency PODEM is introduced ${ }^{14}$. This algorithm is employed to generate automatic test patterns and also expanded the binary decision tree around primary input variables. This algorithm also has sub routine to check the existence of D frontier. Backtracking is also explained in this work using PODEM algorithm. The defect level on behalf of faults is determined by means of a Function of Fault Coverage as well as production. With the help of the definition, the number of stuck-at-faults that are tested as $\mathrm{m}$ and divided with the entire amount of stuck-at-faults $n$ is called as the fault coverage $\mathrm{T}$. The defect level equation as well as the fault coverage is interrelated proportionally with one another which is the main issue.

8. This paper ${ }^{13}$ integrates various algorithms as well as methods regarding the test generation within a unified system that proposed tests on behalf of un-partitioned LSSD logic structures of up to 50,000 logic gates. This paper presents the designing concept in creating the unified system by means of results achieved upon huge logical structures. The VLSI LSSD logic structures achieve the feasible test generation ability as shown in the outcomes of the test generation system.

9. The redundant circuit logic is identified and the implementations of various circuits are compared by generating the circuit test patterns by Automatic-test-pattern generation algorithms. Operative test is impractical for larger circuits and E-beam testing was very expensive. To overcome this inefficiency PODEM $^{16}$ was introduced PODEM expanded the binary decision tree around primary input variables only, thus accelerating the algorithm. PODEM algorithm had a subroutine to check the existence of D-frontier. If not, PODEM backtracks immediately, thus speeding up the process. Backtracking is an important method introduced by Goel ${ }^{13}$ in PODEM.

10. A deficiency model which is used within the computerized circuits ${ }^{17}$ is called as Single stuck line. A line or a hub that is stuck at logical high or low within the computerized circuit is expected by the model. Whenever a line is stuck, it is known as fault. The reliability is enhanced by weighing the required overhead and the fault tolerance is achieved by introducing the redundancy within the digital circuits. A novel design is achieved that simulates the fault on behalf of a digital combinational circuit and it is constructed from 10 gates and 3 inputs using 1 output. $\mathrm{C}$ language is implemented to write the code and outcomes of the single stuck at all faults are presented in detail. Using 10 gates, 3 inputs and 1 output, the implementation of deductive fault simulator is done on behalf of the digital circuit and moreover it implemented and tested.

\section{PROPOSED METHODOLOGY}

The proposed method used for fault detection and fault location is path-oriented decision-making algorithm(PODEM). The first step is effective testing and diagnosing of faults to be carried out in FinFet circuits. For this purpose, the designing of NAND, NOT, NOR gates are done in LTSPICE using FinFET Models(20nm/32nm) and then creating a net list for circuit diagram and later importing into MATLAB in which panara Code is implemented. to apply sensitive test ve Start d locates the fault for both detectable and non-aectecable test case(Ref. Flow Chant 1) and anoliod on Toct tircuit (Fio

Importing FINFET Library into LTSPICE

Estimation of FINFET characteristics of NAND, NOR \& NAND gate

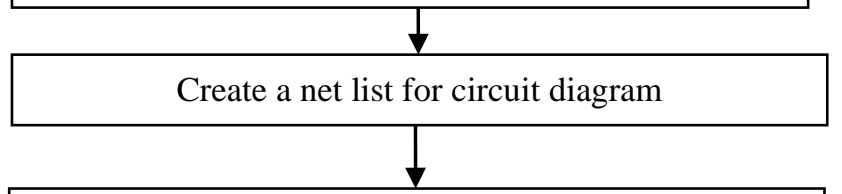

Fault Detection and Location using PODEM

\subsection{Design of Gates}

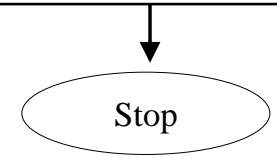

\subsubsection{NAND GATE:}

The design of NAND gate using FinFET models as shown in the below figure. 2, shows that V1, V2, V3 are considered as $1 \mathrm{~V}$. V1 and V2 are considered as input voltage and V3 is used as power supply to FinFET model. VTC curve and delay calculations is a plot of input vs output. The graph of the transfer curve needs to be plotted. The Vth and Vih VIL, $\mathrm{VOH}, \mathrm{VOL}$ all these simulation parameters along with the noise margin high $(\mathrm{NMH}=|\mathrm{VOH}-\mathrm{VIH}|)$ as well as noise margins low $(\mathrm{NML}=|\mathrm{VIL}-\mathrm{VOL}|)$ are calculated and for NOR and NOT gates also results are given (Ref.Table-1)

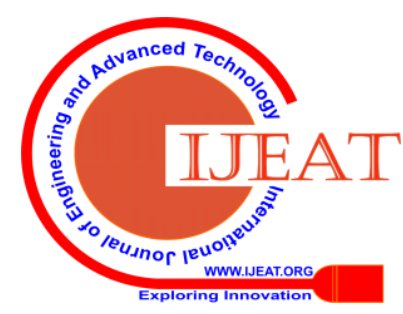




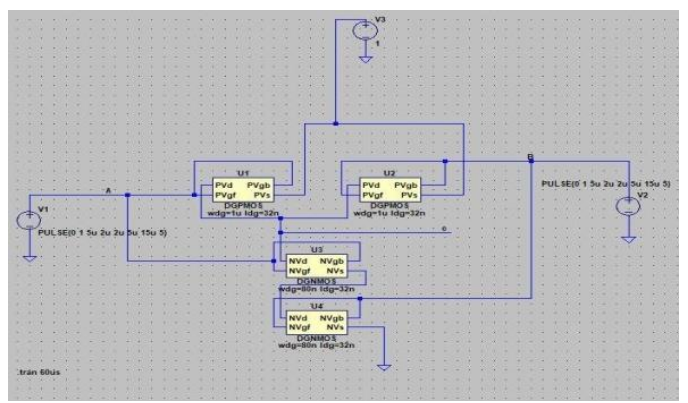

Fig 2. NAND gate design using FinFET library

After the design of the gates these gates are constructed together to find out the fault diagnosis (Refer Fig 3)

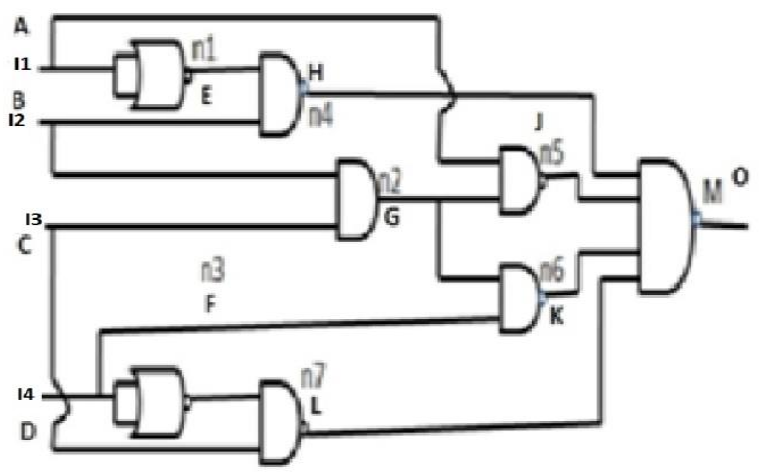

Figure3: Podem Algorithm Test Circuit

\section{NAND GATE:}

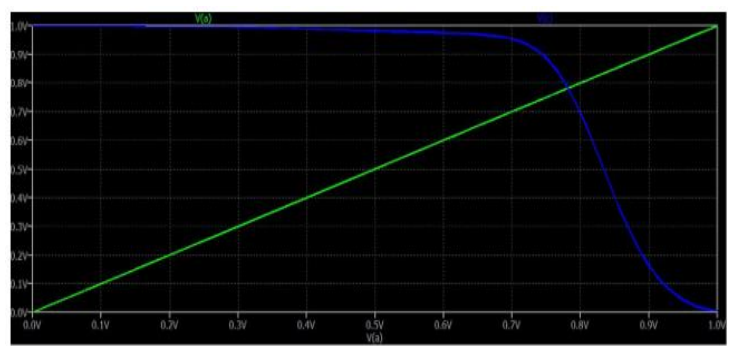

Fig 4: Transfer Characteristics curve f NAND gate. From the above figure the parameters obtained are,

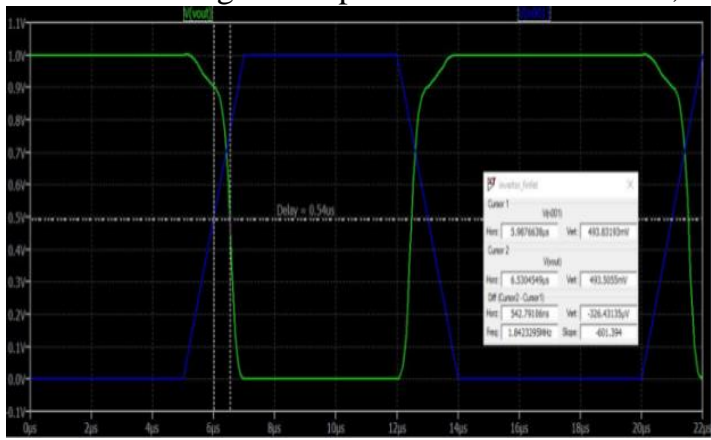

Fig 5: Delay Characteristics of NAND gate

The above fig shows the FinFET NAND gate's delay characteristics. The steady state values obtained are, High to low propagation delay (tpHL): Time is calculated which takes to fall from $\mathrm{VOH}$ to $50 \%$ Low to high propagation delay (tpLH): Time is calculated which takes to rise from $50 \%$ to VOL Propagation delay is calculated as (tp) $=(\mathrm{tpHL}$ $+\mathrm{tpLH}) / 2=0.67 \mu \mathrm{S}$

\section{GATE RESULTS}

GATE Results are given below (Table-1)

\begin{tabular}{|c|c|c|}
\hline [1] NAND & [2] NOR & [3] NOT \\
\hline [4] $\mathrm{V}_{\mathrm{OH}}=0.9 \mathrm{~V}$ & [5] $\mathrm{V}_{\mathrm{OH}}=0.97 \mathrm{~V}$ & [6] $\mathrm{V}_{\mathrm{OH}}=0.8 \mathrm{~V}$ \\
\hline [7] $\mathrm{V}_{\mathrm{OL}}=1 \mathrm{~V}$ & {$[8] \quad \mathrm{V}_{\mathrm{OL}}=0.07 \mathrm{~V}$} & [9] $\mathrm{V}_{\mathrm{OL}}=0.66 \mathrm{~V}$ \\
\hline $\begin{array}{c}\text { [10] } \mathrm{NMH}= \\
|\mathrm{VOH}-\mathrm{VIH}| \\
=0.02 \mathrm{~V}\end{array}$ & $\begin{array}{c}\text { [11] NMH }= \\
|\mathrm{VOH}-\mathrm{VIH}| \\
=0.25 \mathrm{~V}\end{array}$ & $\begin{array}{c}\text { [12] } \mathrm{NMH}= \\
|\mathrm{VOH}-\mathrm{VIH}| \\
=0.07 \mathrm{~V}\end{array}$ \\
\hline $\begin{array}{c}13] \mathrm{NML}=\mid \mathrm{VIL} \\
-\mathrm{VOL} \mid= \\
0.64 \mathrm{~V}\end{array}$ & $\begin{array}{c}14] \mathrm{NML}=\mid \mathrm{VIL} \\
-\mathrm{VOL} \mid= \\
0.513 \mathrm{~V}\end{array}$ & $\begin{array}{c}15] \mathrm{NML}=\mid \mathrm{VIL} \\
-\mathrm{VOL} \mid= \\
0.56 \mathrm{~V}\end{array}$ \\
\hline $\begin{array}{l}\text { [16] Propagation } \\
\text { Delay (tp): } \\
\text { (tpHL + } \\
\text { tpLH)/2 = } \\
0.67 \mu \mathrm{s} \text {. }\end{array}$ & $\begin{array}{l}\text { [17] Propagation } \\
\text { Delay (tp): } \\
\text { (tpHL } \\
\text { tpLH)/2 = } \\
0.04 \mu \mathrm{s} \text {. }\end{array}$ & $\begin{array}{l}\text { [18] Propagation } \\
\text { Delay } \\
\text { (tp):(tpHL + } \\
\text { tpLH)/2 = } \\
0.54 \mu \mathrm{s} .\end{array}$ \\
\hline
\end{tabular}

\section{1 .PODEM (Path-Oriented Decision Making) ALGORITHM IMPLIMENTATION}

An Automatic Test Pattern Generation (ATPG) algorithm is called as PODEM (Path-Oriented Decision Making). The aim of this project is to implement the PODEM algorithm for generating test vectors on behalf of a given fault. Given a circuit and a fault location this algorithm will tell us whether the fault can be detected and a combination of input values which can detect the fault. Whenever one exists, the output resembles a test otherwise it resembles that it is an untestable fault.

\section{Assumptions:}

1. Single stuck-at fault.

2. Written code assuming only 4 types of gates in net list but can be easily extended to other type of gates.

\subsection{Input output descriptions:}

Net list. Txt is the input file having description of nodes in spice net list format. The net list

We used in worked out example:

not1 n1 in1 and2_1 n2 in2 in3 not2 n3 in4 nand2_1 n4 in2 n1 nand2_2 n5 in1 n2 nand2_3 n6 n2 in4 nand2_4 n7 n3 in3 nand4_1 out1 n4 n5 n6 n7

in1, in2, in3 and in 4 are the primary inputs. Out1 is the primary output. Our code can run for any such spice net list (for any number of inputs and any number of outputs)

Fault Location is the node where fault is to be checked, values should be taken from the net list files e.g. n1, n4. Fault Value is the complementary value of the stuck at fault.

"Fault is detected, Input vectors are:" will be the output in case given fault can be sensitized and propagated. "Fault is not detectable" will the output if either fault cannot be sensitized or cannot be propagated or both.

$\mathrm{NCV}=[1,0,1,0,1,-5,-5,1]$ matrix for non-controlling value for each gate type

\subsubsection{PODEM (Path-Oriented Decision Making)}

PODEM is an Automatic Test pattern (ATPG) Algorithm and it works in two major steps:

1. Sensitize the fault

2. Fault propagation 
In the first step, appropriate PI (primary inputs) test are evaluated which excites the fault, that is, causes the complementary value to appear at the fault site

If this is found, then the circuit should be able to propagate the fault, for it to be detectable. In the second step, nodes that are connected to the output gate are assigned non controlling values. One sample circuit is chosen for verification purposes. Various subroutines of the PODEM algorithm are individually verified and explained in the below. Generic steps followed in algorithm:

1. Apply fault excitation conditions.

2. Implement the previous assignment effects.

3. Justify the remained unjustified lines whenever, one or more primary outputs are reached by the fault symptoms. In case that the justification is failed, back trace and go to Step 2.

4 Perform the resulting implications and go to step 2.

6. Once back tracing is completed, to propagate the fault, assign non-controlling values to nodes at output gate (by backtracking and assigning PIs appropriately)

In step 6, we always select a primary input (specifically for PODEM Algorithm). The process in which a suitable primary input is identified and assigns a value to it is known as back trace. Back trace procedure is given with an objective of an internal line in addition to a desired value intended for it and a path backwards is traced in the circuit until an input is found in PODEM and moreover it proved as the most effective over the D-ALG since its search space is limited towards the circuit's Primary Inputs (PIs). However, a search space is present in a D-ALG which includes the entire nodes within the circuit as well as the PIs.

The major modules of the MATLAB code are:

- Podem

- Main Podem

- Read net list

- Convert net list

- Create device map

- Cnctd devices

- Objective

- Back trace

- Imply

- Type

- Type imply

- Gates

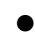

4.2.2 BRIEF FUNCTIONALITY OF EACH MODULE

\section{Podem:}

Global variable declaration. Input assignment and calling function Main Podem

\section{.2. Main Podem:}

Input net list by calling "read net list" and "convert net list" Sensitize the fault

1. There are three major functions used here - objective, back trace and imply. Given a Fault Location, Fault Value pair, it back traces, and evaluates and updates values at each node

Fault propagation

1. Non-controlling value (NCV) is found for each node connected to output node.
2. Objective is called to set each of these nodes to this value. If a set of PI s is found which give NCV values at each of these nodes, ,Fault is detected ${ }^{\text {ee }}$ is displayed along with the set of PI s

\subsubsection{Read net list and convert net list}

These functions read the spice net list, store the input and output nodes in format: [Device ID, Gate Type, Output node, Input Nodes]

Device ID is a unique number assigned to each gate using node map function. Gate Type is a number assigned to a particular type of gate as follows: (Ref: Table -2 \&3)

Table 2

\begin{tabular}{|c|c|c|c|}
\hline Type of gate & Gate type & Type of gate & Gate type \\
\hline NOT & 1 & NAND 2-input & 5 \\
\hline OR 2-input & 2 & XOR 2-input & 6 \\
\hline AND 2-input & 3 & XNOR 2-input & 7 \\
\hline NOR 2-input & 4 & NAND 4- input & 8 \\
\hline
\end{tabular}

*Have used only 4 gates - not, and, Nand, Nand 4 in the code.

4 .objective:

Input to this function is a particular $\mathrm{nG}, \mathrm{nV}$ where $\mathrm{nV}$ is the required value at the node $\mathrm{nG}$. It calls back trace function and back traces this pair of $(\mathrm{nG}, \mathrm{nV})$ till a primary input is achieved and isPI flag is asserted. After each back trace operation, value of $\mathrm{nG}, \mathrm{nV}$ is updated to the next value in stack G.

\section{Back trace:}

Function is called inside objective. Takes an input pair $(\mathrm{nG}, \mathrm{nV})$. In every row of net list it checks where this $\mathrm{nG}$ is an output node of a gate. For this row, according to type of gate and $\mathrm{nV}$, it evaluates the values of inputs of that gate using "type" function. These values of inputs to the gate are stored in a stack $\mathrm{G}$ and stack $\mathrm{V}$ variable.

\section{Type:}

Used in backtracking. Takes a row of format device ID, gate type, output nodes values, and input nodes values. Checks the type of gate and calls appropriate gate function (say Nand out for gate type $=5$ for Nand gate) and returns the row with updated input values.

\section{Imply:}

The main objective of this operation is performing one logic simulation proceeding from the circuit depending upon the primary Input values. The inputs are (PI, PI Value) obtained from the objective function. It searches for the gates in net list where PI node is an input and stores them in device connected array. Then it calls "imply device" function which operates as: It calls "type imply" function which evaluates the output node values. Also updates the node values array. This runs in a while loop which runs till all primary outputs are implied.

\section{Type imply:}

Similar to type function but it is used in imply. According to the type, corresponding gates are called. Difference here is that inputs are known and output is to be implied. The updated row is output from the function. 


\section{Gates:}

\section{NAND OUT:}

Function for 2 input NAND gate. Inputs to this function are the two inputs and output of the row which is to be updated and g. $\mathrm{g}$ will be 0 for backtracking and $\mathrm{g}=1$ for implying.

\section{AND OUT:}

Similar to nand out. Nand 4input:

Similar to nand out.

\section{NOT_OUT:}

Similar to nand out. Worked out examples:

We have worked out the algorithm on the following circuit. Codes in MATLAB and python are in the code-and-demo folder.

\section{Example:}

Net list: Alphabets are shown here just to facilitate understanding of below diagram not1 $\mathrm{n} 1(\mathrm{E})$ in1(A)

$$
\text { and2_1 n2 (G) in2 (B) in3(C) }
$$

not2 n3 (F) in4 (D) nand2_1 n4 (H) in2 n1 nand2_2 n5 (J) n1 n2 nand2_3 n6 (K) n2 in4

nand2_4 n7 (L) n3 in3 nand4_1 out1 (M) n4 n5 n6 n7 using node map function all nodes of net list will be assigned a unique device ID as given in Table-3

Table 3for results

\begin{tabular}{|c|l|l|}
\hline Device ID & Nodes from Figure & Node of net list \\
\hline 1 & E & n1 \\
\hline 2 & A & in1 \\
\hline 3 & G & n2 \\
\hline 4 & B & in2 \\
\hline 5 & C & in3 \\
\hline 6 & F & n3 \\
\hline 7 & D & in4 \\
\hline 8 & H & n4 \\
\hline 9 & J & n5 \\
\hline 10 & K & n6 \\
\hline 11 & L & n7 \\
\hline 12 & M & out1 \\
\hline
\end{tabular}

\section{RESULTS AND DISCUSSIONS}

\section{After running MATLAB CODE on Test CIRCUIT}

1. Fault at node K i.e. stuck-at $-1=>\mathrm{V}=0$ :

Step 1: Set objective $(K, 0)$ and back trace $(K, 0)$ which gives $(G, 1)$ and $(D, 1) . D=1$ found by backtracking (till a primary input). Now call imply $(\mathrm{D}, 1)$

$\mathrm{D}=1$ implies $\mathrm{F}=0$ implies $\mathrm{L}=1$ while other nodes are still unassigned

Step 2: Now check for value at fault location. It is still unassigned (value $=-5$ ), so continue with using the second option of back trace $(K, 0)$. i.e. $(G, 1)$ (as stored in stack earlier) . Back trace $(G, 1)$ gives $(B, 1)$ and $(C, 1)$.

Imply with $(B, 1)$ gives $G=-5, K=-5$

Step 3: Now imply with $C=1 .=>G=1, K=0$. So fault is sensitized. Step 4: For propagation, back trace $(\mathrm{J}, 1)=>\mathrm{A}=0$, $\mathrm{E}=1, \mathrm{H}=0$.
Step 5: Since $\mathrm{H}=0$, fault cannot be propagated. So no test exists. And fault is not detectable 2 Fault at node K, stuck-at$0=>\mathrm{V}=1$

Step 1: Set objective $(K, 1)$. Back trace $(K, 1)=>D=0$. Imply $(\mathrm{D}, 0)$ will give $\mathrm{F}=1, \mathrm{~K}=1$.Fault at $\mathrm{K}$ is sensitized

Step 2: To propagate the fault, back trace $(\mathrm{H}, 1)=>(\mathrm{E}, 0)$ $=>(\mathrm{A}, 1),(\mathrm{B}, \mathrm{X})$

Imply $(A, 1)=>$ no changes in node values.

Step 3: back trace $(\mathrm{J}, 1)=>(\mathrm{G}, 0)=>(\mathrm{C}, 0) \operatorname{imply}(\mathrm{C}, 0)=>$ $(\mathrm{J}, 1),(\mathrm{L}, 1)$

Step 4: Since J, L and $\mathrm{H}$, all are set to Non-controlling value of Nand4. Fault can be propagated. Input Vector is (A, $\mathrm{B}, \mathrm{C}, \mathrm{D})=(1, \mathrm{X}, 0,0)$

Proposed test algorithm that provides better diagnosing of faults in FinFET circuits

\section{for the given test circuit}

\subsection{PODEM ALGORITHM RESULT:}

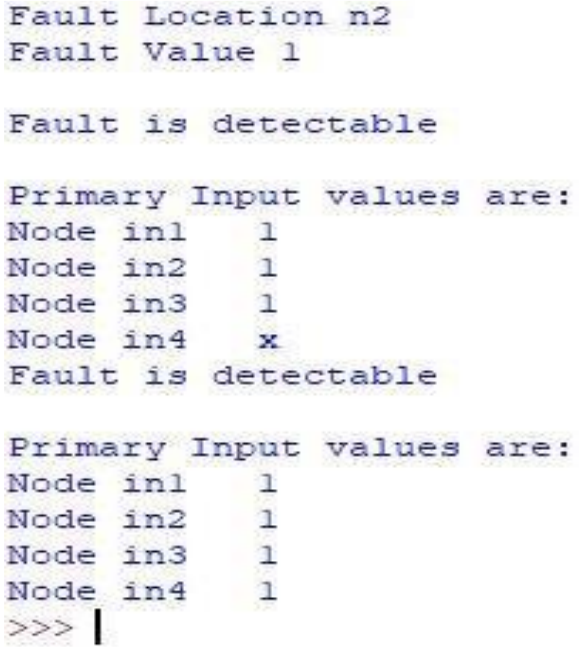

Figure6 : Fault value is Detectable

Figure7: Fault value is not Detectable

PODEM Algorithm for the Test Circuit (Ref: Fig 2) the fault is detected at node n4 (Device Id H) and Fault Value is 0, similarly for any given Fin FET based Circuit faults can be detected and location can be identified with sensitive Test Vector at the inputs Ref:Figsd 6 \&7)

\section{CONCLUSION}

In this paper, designing of NAND, NOR and NOT gates are done using FinFet model \& each gate with logic outputs are verified as shown in graphs. The simulation results show that for each gate VTC curves has been plotted and delays, noise margin values (VTh, VIH, VIL, VOH, VOL, NMH, and NML) areobtained. 
The circuit diagram is designed and faults are analyzed, detected and fault location is identified for the given circuit. Sensitive input Test Vectors are identified for both Fault detectable and not Detectable conditions (Ref: 6 \& 7) By using PODEM algorithm, fault location and faults are obtained, best vector selection is found. Furthermore, In this Research work it is attempted application of PODEM Algorithm concept to detect and find fault location in advanced 20nm/32nm FinFET based Combinational Circuits for the first time and it is proved.

\section{ACKNOWLEDGEMENTS}

Authors would like to thank Shri B H V S N Murthy, DS\&Director, RCI and Dr Bheema Rao, HOD, ECE Dept. and also DRC members NITW for their constant encouragement, valuable suggestions and support for carrying out this work as part of my $\mathrm{PhD}$ work

\section{REFERENCES}

1. Thakker, R. A., Sathe, C., Sachid, A. B., Baghini, M. S., Rao, V. R., \& Patil, M. B. (2009). A novel table-based approach for design of FinFET circuits. IEEE Transactions on Computer-Aided Design of Integrated Circuits and Systems, 28(7), 1061-1070.

2. Gu,J.,Keane,J.,Sapatnekar,S.,\&Kim,C.(2006,September).Width quantization aware FinFET circuit design. In Custom Integrated Circuits Conference, 2006. CICC'06.IEEE (pp.337-340).IEEE

3. Bhardwaj, N., Mahor, V., \&Pattanaik, M. (2015, November).RobustFinFET based highly noise immune power gated SRAM circuit design. In Communication Networks (ICCN), 2015 international Conference on (pp.310-316). IEEE.

4. Hajare, R.Lakshminarayana, C.,Sumanth ,S.C.,\&Anish, A.R.(2015,December) .Design and evaluation of FinFET based digital circuits for high speed ICs .In Emerging Research in Electronics, Computer Science and Technology ICERECT), 2015 International Conference on (pp.162-167). IEEE.

5. M.O.Simsir, A.Bhoj and N.K.Jha, "Fault modelling for FinFET circuits,"2010IEEE/ACM International Symposium on Nano scale Architectures, Anaheim, CA, 2010, pp.41-46.

6. R.Wadsack, "Fault modelling and logics imulation of CMS and MS Integrated circuits ,’Bell System TechnicalJ.,vol.57,pp.1449-1474,May 1978

7. Harutyunyan, G., Tshagharyan, G., \&Zorian, Y. (2015, April). Impact of parameter variations on FinFET faults. In VLSI Test Symposium (VTS), 2015 IEEE33rd (pp.1-4).IEEE

8. G.Harutyunyan, G.Tshagharyan, V.VardanianandY.Zorian," Fault modelling and test algorithm creation strategy for FinFET-based memories," 2014 IEEE3 $2^{\text {nd }}$ VLSI Test Symposium (VTS), Napa, CA, 2014, pp.1-6.

9. Musala, S., \& Srinivasulu, A.(2016,April).Self-testing and fault secure XOR/XNOR circuit using FinFETs. In Communication and Signal Processing (ICCSP), 2016 International Conference on (pp.1222-1226).IEEE.

10. S.Yin, X.ZhuandO.Kaynak,"ImprovedPLSFoc used on KeyPerformance-Indicator- Related Fault Diagnosis, "in IEEE Transaction son Industrial Electronics, vol.62,no.3,pp.1651 1658,March201

11. Kuan-YingChiang; Yu-HaoHo; Yo-Wei Chen; Cheng-Sheng Pan; James Chien-MoLi, Fault Simulation and Test Pattern Generation for Cross-Gate Defects in FinFET Circuits, 2015IEEE 24th Asian Test Symposium (ATS), 2015.

12. M.O.Simsir, A.Bhoj and N.K.Jha,"Fault modelling for FinFET circuits, "2010 IEEE/ACM International Symposium on Nano scale Architectures, Anaheim, CA,2010,pp.41-46.

13. P.Goel and B. Rosales, "PODEM-X: An Automatic Test Generation System for VLSl Structures," Proc. DesignAutomation Conf., June 1981, pp. 260-268

14. S. Funatsu and M. Kawai, "An Automatic Test-Generation System for Large Digital Circuits, ” IEEE Design \&Test of Computers, Oct. 1985, pp. 54-60.

15. Gong, L., Hu, H., \& Zhang, Y. Test Generation for Sequential Circuit Using PODEM Algorithm.
16. Varadharajaperumal, M. Path Oriented Decision Making (PODEM).

17. Ahmed K. Jameil, "a new single stuck fault detection algorithm for digital circuits "International Journal Of Engineering Research and General Science Volume 3, Issue · January 2015.

18. K.Praveen Kumar, Kumaraswamy Gajula "Fractal Array antenna Design for C-Band Applications", International Journal of Innovative Technology and Exploring Engineering (IJITEE), Volume-8 Issue-8 June, 2019.

19. K.Praveen Kumar, "Active Switchable Band-Notched UWB Patch Antenna", International Journal of Innovative Technology and Exploring Engineering (IJITEE), Volume-8 Issue-8 June, 2019.

20. K.Praveen Kumar, "Circularly Polarization of Edge-Fed Square Patch Antenna using Truncated Technique for WLAN Applications", International Journal of Innovative Technology and Exploring Engineering (IJITEE), Volume-8 Issue-8 June, 2019.

\section{AUTHOR PROFILE}

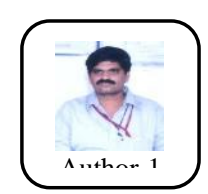

K V B V Rayudu graduated from Institution of Electronics and telecommunication Engineers (IETE), New Delhi during Dec 1990 and obtained MS Centre Imarat (RCI), DRDO, Hyderabad as Scientist in R\&QA activities of Missile Systems. Contributed significantly in Parts Management, Qualification, Testing, Failure Analysis, reliability Analysis \& Screening Policy of Electronic Components \& Systems for Aerospace Applications. Planned and Played Key role for ISO 9001:2000 Certification and Aerospace Quality Management System AS 9100:2009certification to RCI, Hyderabad. His Research Interests include VLSI Testing, VLSI Fault Simulation, Modelling \& Diagnosis Reliability Analysis, Failure Analysis, Quality Management System Certifications applications of ANN, GA, SVM for optimization etc.

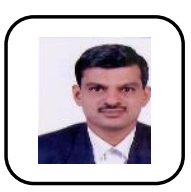

D R Jahagirdar received his B.E. degree in Electronics Engineering in 1990, from Govt. College of Engineering, Amravati University, Maharashtra, India. He received M. Tech. in Microwave Engineering in 1992, from Indian Institute of Technology, Kharagpur, West Bengal, India. He was a Research Assistant at Sponsored Research and Industrial Consultancy at IIT, Kharagpur. Later, he joined Antenna Products Division of Electronics Corporation of India Ltd, Hyderabad. He obtained Ph. D. in 1997 from the Department of Electronics and Computer Science, University of Southampton, UK. He received scholarship from the Commonwealth Scholarship Commission UK to pursue PhD. He joined Research Centre Imarat, DRDO, Hyderabad in May 2000. He has won 'Best Paper Award' at the University of Leeds, UK organized by IEEE UKRI section. He has received Prof. S.K. Mitra memorial award for 'Best research oriented paper' from IETE in 2002. He received Young scientist award at IETE-IRSI International Radar symposium Bangalore in 2005. He also received laboratory scientist of the year award for 2006. He is a Fellow of IETE and senior member of IEEE, Antennas and Propagation Society and Microwave Theory and Techniques Society. He is also a member of URSI. Recently he has been listed in Marquis' Who's who in he world. His area of interest is microwave antennas and arrays for radars.

Second Author profile which contains their education details, their publications, research work, membership, achievements, with photo that will be maximum 200-400 words.

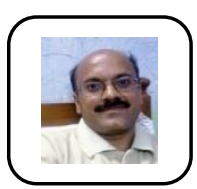

Dr.Sriharirao Patri is working as Assoc Prof at NITWarangal in the dept.of ECE. His Research intrests include RFIC Design, VLSI Testing, Fault Diagnosis Analog/digital IC design, DSP Architecture, Analog LDO's. He has published 10 technical papers in Reputed international journals/Presented in Conferences. He has conducted various cour in VLSI area and guided 5PhD Students. 